lieve it worthy of the most careful consideration of every physician employing anesthetics.

No doubt M.S. possesses great advantages over pure chloroform or sulphuric ether and is an excellent anesthetic, but in the light of the presumed correctness of Schleich's reasoning, outlined above, M.S. or any other combination of anesthetic drugs, the boiling point of which does not correspond to the temperature of the human body, can not be considered as safe as anesthol.

The improvement in general anesthesia brought about within the last few years has been manifold. Primary administration of nitrous oxid, followed by ether oxygen anesthesia, carried out by expert anesthetists such as New York may well be proud of, means a great step forward in the right direction. The same is true in regard to the efforts on the part of the various staffs of attending physicians and surgeons of our large hospitals, to create a paid position for permanent narcotizers. No doubt the future will see such experts in place in every well-conducted hospital, and thus put a stop to the jeopardizing of the lives of our patients by the inveterate method of having the youngest doctor, fresh from college, untrained and inexperienced as he is, attend to narcoses.

However, in private practice and in places remote from the larger cities, such expert anesthetizers can not always be at our disposal. The country doctor must carry the anesthetic in his valise; he can not regularly employ a second man, who carries with him the cylinder's filled with nitrous oxid, nor can he himself bring along the large though ingenious and useful rubber bags, enabling the doctor to administer ether in a better and more scientific way than is possible with the paper and towel cone.

Here simpler appliances with regard to mask as. well as the anesthetic are needed. Ether and chloroform administered with the help of uncomplicated masks or cones have carried the field so far. Anesthol, as has been demonstrated above, being a true solution, not a mixture, may thus well be employed in these cases in place of the former certainly more injurious pure drugs.

I should be pleased, indeed, if these lines should induce others to try anesthol, and they should get from their patients so gratifying a description of their subjective impression as I recently received from a colleague, on whom I operated at his home three times in close succession for a rapidly spreading phlegmon of hand and arm resulting from a finger infection. In each instance the anesthesia was conducted with anesthol preceded by a hypodermic of $1 / 4$ of morphin. His words to me after this threefold experience were these:

"You take it (anesthol) without knowing what you are taking, and when you awake you do not know what you have taken. It does not annoy you; it does not make you sick. It is simply a question of falling asleep and waking up."

The Need of Organization.-Dr. James C. Johnston, in the Philadelphia Medical Journal, says: "The worst of the many evils with which the medical profession has to contend is one of which all our national experience has taught us the folly, decentralization. It is evident throughout. Its business is worse handled than any other. Instead of trusts, in which overproduction is restrained, in which wages can be fixed and maintained, and in which the incompetent and superfluous are eliminated, it is every man for himself and the devil and his colleagues for the hindmost. This is true of its material for investigation and of the journals it supports, as well as actual practice."

\section{FEVERS OF DOUBTFUL NATURE IN INFANCY.} PRELIMINARY CLINICAL NOTE.

\section{J. L. DUENAS, M.D.}

Attending Physiclan to the Children's Ward, Hospital No. 1; VisitIng Physician of the Training School for Girls of Cuba; Children's Physician in Tamayo Dispensary. havana, cuba.

In the island of Cuba the febrile process is found with extraordinary frequency during infancy and childhood. The meaning of the fever symptom in a tropical country where typhoid fever, malaria, influenza and yellow fever exist in all their forms and varieties has always been a matter of great local interest which has given rise to lively discussions. Not over 20 years ago the diagnosis of febrile diseases was made by the methods of the old clinic, and the anatomic denominations were the ones which prevailed in the nosologic charts.

\section{ALL FEVERS CONSIDERED MALARIAL.}

At the time that little was believed in the existence of typhoid fever in this island, and when it was supposed that yellow fever only attacked unacclimated foreigners, the tendency of physicians was to diagnose as malaria all cases where a local cause to the process could not be easily found, or where it could not be referred to another well-defined general disease.

During the decade of 1880 to 1890 the existence of typhoid fever in our towns of importance was well demonstrated pathologically; but notwithstanding many persisted in denying it, continuing to classify as malaria all cases of remittent fever of longer or shorter duration. A true epidemic of malaria-mania spread itself among the physicians to such an extent that seldom a febrile process was not attributed to malaria, even when there were evident signs to explain its origin in a different way. At about that time works were published in other countries intended to exhibit the different forms and localizations of this infection of the blood.

In 1890 I presented to the First Cuban Medical Congress a paper on the use of quinin in Havana, where I tried to refute the tendency to abuse of that alkaloid, particularly in children, and I firmly denied the existence of so many cases of malaria, either as a primary disease or as a secondary process, always supposed to be associated to many other pathologic processes. A very small number of physicians supported my idea at that time.

\section{THE VARIOUS OTHER CAUSES OF FEVER.}

In a lecture which I delivered two years later to the "Sociedad de Estudios Clinicos," I initiated a discussion on the several 'kinds of fever reigning in this city, especially during the hot season, and which attacked children with preference, being then inclined to admit for many of them an infectious cause of intestinal origin. I tried to differentiate them from typhoid fever by their etiology, their symptoms and their course, making saprophytism and endogenic or exogenic autointoxication responsible for some of our types of tropical fevers. I called attention to the cases of typhoid fever in which autopsy did not show the characteristic lesions of Peyer's patches and only the isolated follicles of the large intestine were involved, for which reason these cases were denominated colo-typhus by the ancient writers. I pointed out besides the coincidence of infectious enteritis in infancy during the epidemic of those fevers in children of all ages and young adults, considering coloinfection, as the physicians of Lyon's school did, as an attenuated expression of Eberth-infection.

In 1893, and as a consequence of a dedication to the study of children's diseases, I presented to the First 
Pan-American Medical Congress, held at Washington, a paper on "Primary Acute Entero-colitis in Infancy," in which $I$ described the different varieties of types of gastrointestinal troubles in the first years of life, and I called attention to the frequency and predominance among us of the eclamptic, cerebral, gastric and typhoid forms of the ancients in opposition to the cholera infantum which was common in the United States and certain regions of Europe. In that work I demonstrated with facts taken from my personal experience that in that division of pediatrics I had not observed anything in Havana which had not been minutely described in other countries by the old masters of that branch of medicine.

\section{THE INFREQUENCE OF MALARIA.}

In the course of recent years it has been proved beyond doubt by means of the observation of the blood that malaria is not frequent in Havana, as I affirmed in 1890 , based on the clinical and epidemiologic observation. It has also been proved that the anopheles species of mosquito is seldom found within the radius of the city, being somewhat frequent in the suburbs and much more in the country where cases of acute and chronic malaria are common.

The results of modern scientific investigations have thus brought about a change in the ideas regarding the nature of our fevers. To-day it is accepted in a general way that malaria is not so frequent in Havana; that typhoid fever exists in greater proportion and that, on the other hand, there is a large number of febrile patients in which neither malaria nor typhoid fever can be diagnosed, not only on account of the clinical signs, but also by the constant negative results of the hematologic analysis, as far as Laveran's parasites and agglutination tests are concerned.

The fact that the Island of Cuba is one of the main foci of yellow fever introduces a new element of discussion in the etiology and diagnosis of infantile fevers, inasmuch as clinical and epidemiologic demonstrations have clearly shown the receptivity of the natives, denied for such a long time, for that dreadful disease.

\section{THE DIAGNOSIS COMPLICATED.}

I have thought it necessary to make this historical sketch in order to show how divided the opinion of physicians about the nature of our tropical fevers has always been; also to point out the difficulties surrounding the diagnosis of infantile pyrexias in a country where malaria, typhoid fever, influenza and yellow fever exist in their usual forms of epidemic exacerbations, and, where it is necessary to admit at the same time the existence of fevers as yet undeterminated. and which have their origin in the digestive tube and which the dereloping modern science accepts in all other countries since the intestinal autointoxication doctrine was established to explain the origin of general and organic troubles similar to those produced by other better known specific infections.

\section{THE GASTROINTESTINAL FEVER.}

The group of fevers which I intend to describe here constitute two principal types, one of rapid, the other of slow evolution. Both are common to all periods of infancy and childhood, but the younger the child the more frequently the rapid evolutive forms occur. The first type is characterized by feverish accesses most commonly of a depressive rather than of an excitative nature. The disease in many cases attacks the child unawares when he is in excellent state of health; but it is frequently preceded by digestive derangements, diarrhea and simple indigestion, the gastrointestinal symptoms being always more or less apparent. At first vomiting and constipation may exist or yellowish, greenish, thick mucous stools accompanied by redness and irritation of the anus. The tongue is slightly coated and if the child is traversing the dental period stomatitis and throat inflammation may exist to a certain degree. In some cases the symptoms mentioned do not exist, the only phenomena apparent being certain sensibility experienced on pressure of the abdomen, somewhat intensified on the region of the liver and accompanied by muscular contractions.

From the first stage the temperature rises to 104 or more. During the first day of the disease the thermic cycle is sustained or continuous, or interrupted by slight descents followed by new rises. During the succeeding days the temperature follows a different course dependent on the evolutions taking place in the morbid process of the disease, whether in the direction of recovery or death. In the first case the fever assumes a remittent character and in a few days completely disappears together with all other symptoms which gradually disappear at the same time. When the process of the disease must certainly end in death the temperature has a marked tendency to continuity, the cerebral symptoms grow more intensified, convulsive phenomena appear accompanied by coldness of the extremities and suppression of the urine. The little patient may die in the course of 24,36 or 48 hours.

The causes which in Cuba have always originated discussions on the nature of these cases are:

1. The belief in the prevalence of endemic paludic germs.

2. The possibility existing at present that they may constitute syderant types of yellow fever.

3 . The absence of phenomena in the digestive apparatus frequently observed in the early period of invasion of the disease.

In other countries the differential diagnosis of these cases is made on malarial infections, typhoid infections, acute cases of influenza, cerebrospinal meningitis, primary tubercular meningitis, or secondary meningitis of rapid evolution; but in all of them the influence of intestinal infection on the origin of these pathologic cases which are so frequent during the first stages of life, is to-day recognized.

Marfan, Saint Philip, Comby, Escherich, Bagınsky, in Europe; Booker, Jacobi, Starr, Holt, Crandall, and many others, in America, have described the rapid type of these intestinal infections. Modern doctrines compel us to classify these cases as fevers whose nature is still undergoing due study and investigation, so as to bring them to the rank of definitely specified infections. But they correspond exactly to the severe eclamptic, cerebral, meningitic or convulsive forms of acute enteritis, or enterocolitis which are described by such authors of old. text books, as: Bouchut, West, Trousseau, Rilliez and Barthez, Meigs, who pointed them out as a very frequent ocurrence during the first two years of life. The diseases arc the same. The only difference to be observed is in the denomination which varies in accordance with modern theories held as to its etiology and pathogenic mechanism.

REASONS FOR THE GASTROINTESTINAL FEVER THEORY.

In the Island of Cuba, and above all, in the City of Havana and other localities where malaria is not so frequent, I can assure, after more than twenty years' experience, that the majority of these cases of acute infec- 
tions have in infancy a gastrointestinal origin owing to the following reasons :

1. The etiologic investigation allows us to discover among the antecedents of the case, more or less serious transgressions in the dietary hygiene, on the strict observance of which $I$ have greatly insisted in my work on acute enterocolitis, published in the "Transactions of the first Pan-American Medical Congress" (Vol. II, p. 1343). I will here add that in many instances the mothers absolutely deny said origin, but clinical observation reveals abnormal discharges. In some cases they confess the fault incurred and later the exact causes of the intestinal derangements can be discovered.

2. The epidemiologic investigation shows, on the other hand, that the disease is more frequent during the warm weather and coincides with the development of fever of typhic nature, with the different types and varieties of gastrointestinal accidents, in many children under two years of age, and with the remittent prolonged cases of fever in children above that age. Its frequent repetition observed in the same child and always obeying to the same cause, dissipates all idea of typhoid or yellow fever; diseases which do not attack the same person twice in accordance with the opinion generally established and admitted.

3. The bacteriologic examination demonstrates the existence of great number of Bacillus coli communis in virulent condition, as well as of other saprophytes in the potable waters of Havana. Very frequently unboiled water is administered to nursing children.

4. The hematologic examination reveals the absence of plasmodies in the blood obtained from these small patients as well as the absence of the serum reaction of Widal.

5. The treatment by means of quinin though administered hypodermically, is absolutely inefficient. The children inevitably die while taking quinin unless use be previously made of intestinal eliminators. The greater number of cures and the rapidity with which they are affected depend evidently on the energetic administration of calomel, castor oil or other laxatives, the use of antiseptics and intestinal injections, stimulants of nutrition, and rigorous hydric diet.

\section{CLINICAL CHARACTERISTICS.}

Very frequently the fever reappears owing to some neglect of the dietetic plan during convalescence and again yields under the same therapeutic treatment. The inadequate, defective or tardy treatment, the lack of compliance with any detail of the medical prescriptions, physical weakness on the part of the child to resist the disease, intensity of the toxemia owing to the retention of toxic substance in the higher portion of the intestinal canal, due to the atony of the muscular coat, hereditary tendencies or renal insufficiency, are all circumstances which contribute to aggravate these cases, originate secondary infections, and occasion the fatal end of the disease.

'The second type of these fevers comprises the cases of slow course. The persistence of fever constitutes the ruling feature of this group. Together with the febrile symptoms gastrointestinal troubles are developed as well as troubles of the other organs without there being any constant relation between the former and the latter.

The disease as a whole reproduces the clinical characteristics of an incomplete typhoid fever, and it may last from one to four weeks, there being cases of longer duration.
The thermic cycle varies greatly: the type of subcontinuous fever is very common during the first and second day, followed by remissions which become more and more pronounced in the subsequent days. There may be accesses every two days and periods of abatements lasting one, two or three days followed by a new rise in temperature which generally occurs together with an aggravation of the intestinal symptoms. Therapeutic intervention directed against the gastrointestinal states always modifies the course of those cases. At other times a moderate and rather regular remittent fever is observed which lasts for many days without any more symptoms until suddenly febrile paroxysms present themselves which then become complicated with cerebral phenomena and may be followed by death with all symptoms of toxemia. In other little patients the fever maintains its remittent character for many days and then diminishes gradually in an irregular way.

The digestive disorders accompanying the feverish syndrome may vary from the mildest form to the predominance of gastrointestinal localization of certain intensity. The tongue is red and somewhat desquamated or slightly coated; there is vomiting of mucous matter, fetid breath, anorexia and thirst; the stools have a semifluid consistency of greenish yellow color, grumous and mucoid, very foul at first and occasionally like those of dysentery. The urine is turbid, loaded with uric acid, urates and sometimes mucin or albumin. Usually the stools turn yellow and lose their offensive smell.

The visceral infarctions are frequent; the liver is almost always augmented and its consistency may reach that which is observed in cirrhosis. The spleen may also be enlarged, but this is less frequent. This fact may occur at the onset of the disease. Finally there are cases where both viscera are in normal state from the beginning to the end of the disease.

The exploration of the abdomen shows pain and distension at the level of the transverse colon in the ascending and descending colon, these symptoms disappearing later on more or less intense fever remaining as the only manifestation of the pathologic process.

Not unfrequently cases are observed which act in a normal way, and with tendency to constipation, but oily laxatives determine the expulsion of mucosities and the attenuation or temporary disappearance of fever. This result at other times can not be obtained without a change in the alimentation.

The type of enteritis of typhoid form of the ancient writers is recognized when studying some of these pathologic classifications in children under two years of age. In other children the morbid process has the type of the gastrointestinal troubles of the ancient writers, or that of gastric fever, which traditional observation separated from the similar form of typhoid fever and malaria.

The diagnostic study of every fever is one of the most difficult ones that may present themselves in pediatric practice. Before the birth of bacteriology the clinical and epidemiologic criterion served as a base to distinguish general diseases among themselves. Often the diagnosis was left uncertain when it could not be confirmed by means of pathologic anatomy. Bacteriologic discoveries have afterward solved many etiologic problems; but doubtless the negative result and the insufficiency of our knowledge in that respect have contributed to a certain extent to throw more confusion on the etiologic conception of some morbid processes. Besides, modern procedures of investigation and experimental demonstrations have revealed that side by side with the better known specific infections there are others which are not 
classified as yet. This has given rise since the notable work of Bouchard, to the doctrine of intestinal autoinfections, by which it is tried to explain at present the nature of certain obscure, incomplete or atypical processes which according to the epidemiologic criterion were systematically included in either of the two great pyrexias, typhoid and malaria, and also in yellow fever in countries like ours. In order, therefore, to properly solve the diagnostic problem we must be more cautious than ever, trying to resort to every possible source of positive information; clinical observation, first, laboratory work afterward and a minute etiologic investigation comprising the patient and the surroundings.

Having thus excluded malaria, grippe or typhoid as origins, disregarding the other processes, local or general, capable of producing fevers of a similar course in children, it may be admitted that the forms of slow course that have been described are, in the same way as those of rapid course, consequences of putrefactions that take place in the intestinal tube. These putrefactions may be caused either by direct introduction of morbid agents with the food or by the increased virulence of the micro-organism habitually living in the intestine in a saprophytic state, due to faults in the dietary or to other external influences. The effects are the same: increased intestinal fermentation, production of toxic element and consecutive autointoxication after their absorption in the circulatory system. The feverish syndrome represents a functional trouble depending on an infection which takes place on the surface of the mucous membrane with. out leaving a trace of any kind. At other times the infection causes more or less extensive injuries which may be propagated to other organs of the digestive apparatus and to the remainder of the organism.

This way of thinking agrees with the work carried out in recent times by Hayen, Lesage, Thiercelin, Escherich, Baginsky, Booker and many others on the part acted by divers micro-organism in the production of infantile gastroenteritis.

An inspection of medical literature shows that the same doubts have arisen in all parts of the world about the classification of those feverish types, which differ in many respects from all the other groups of defined specific infections.

Kelsh and Kiener, Ferée and Vincent in Algiers have argued against those morbid states described by Sesary under the name of gastrointestinal fevers. Legrain. more recently, has attributed them to the colon bacillus and he affirms that this agent may give rise to a whole series of cases of remittent fevers which offer a scale of seriousness from the simple mild form to the syderant form resembling yellow fever. Manson studied them among "unclassified tropical fevers." In Italy, Tomaselli, Cantani, Fazio and others discussed them many years ago as a sui goneris infection. Fedé, of Naples, describes it with the name of "gastrointestinal infection of infancy," and it is very common to find allusions to those diverse types of fevers in the medical literature of the United States.

'The expression "cntero-septic fever" proposed by Dr. Tomás, of Matanzas, has become very usual in Cuba, and it is certainly preferable, as it has the advantage of expressing the location and nature of the pathologic phenomena without making reference to any specific micro-organism.

In conclusion, the feverish process during the infantile period of life is very frequent in the Island of Cuba. In a great number of times fever owes its existence to the same infectious, diseases which have been described by observers of other countries. Ignorance of this fact has been one of the causes which have contributed to mislead medical opinion on the nature of infantile pyrexias. As a rule, these pyrexias have a tendency to reveal a similar clinical expression during early stages of life. In other words, there does not always exist a constant relation between the infectious agent and the clinical phenomena exhibited by the patient. Hence the diagnosis is extremely difficult and requires minute and wide information of a clinical, etiologic, epidemiologic and experimental order to reach with more certainty the scientific truth.

But it can not be denied that most of the infantile fevers come from intestinal infection. These fevers which I have classified in two groups, according to their duration, correspond to the same processes that in the ancient nosologic classifications were called acute enteritis or enterocolitis. and which at present are named by some writers infectious gastroenteritis or infectious enterocolitis; also intestinal fevers or intestinal infections. Endogenic or ectogenic infections of the intestinal tract are the ones which give rise to the feverish types already described; the septicemic or rapid and the enteroseptic or slow, according to the predominance of the symptoms of autointoxication of the organism or whether more or less extensive inflamatory lesions on the mucous membrane of the intestine are produced.

The prognosis in these fevers depends on the virulence of the germs producing them and on the amount of resistence opposed to it by the organism of the child; but to a great extent it is secondary to the opportunity and energy of the treatment employed as well as to the experience acquired on infantile diseases.

\section{A CONTRIBUTION TO THE PATHOLOGIC HIS- TOLOGY OF SYPHILITIC ETHMOIDITIS. \\ J. L. GOODALE, M.D. Bostor.}

Among the causes of chronic inflammation of the ethmoid bone syphilis has long been considered to play a prominent part. In the cases, however, which usually come under observation the process is well developed, and it is a rare experience to be able to follow the condition from the beginning. The following case is, therefore, of particular interest, especially in view of the fact that a histologic examination of the anterior portion of the middle turbinate was made at an early stage of the disease.

\section{NARRATION OF CASE.}

P. J. B., a man 30 years of age, was referred to me by $\mathrm{Dr}$ H. W. Kilburn, Oct, 25,1899 , with reference to a possible source of irritation in the nose, which might cause inflammation in the conjunctiva of the right eye. Examination showed an enlarged right middle turbinate, the nose and throat being otherwise essentially normal in appearance. The patient's previous history was negative.

The anterior end of the right middle turbinate was snared, and found to exhibit cystic enlargement of the bone. Novem. ber 1 , a portion of the stump remaining was removed with the conchotome. November 16 , examination of the right middle turbinate showed an irregular swelling at the margin of the stump which had the appearance of granulation tissue, and which was of such size as to occupy almost the same region as the original anterior extremity. This was removed by the snare. November 21, the granulation tissue had reappeared, and by December 5 had exceeded even the orig. inal size of the turbinate. The patient was then put on protoiodid of mercury, one quarter grain, t.i.d. December 19, examination showed the growth to have become smaller, and 